\title{
Nuevos dispositivos electrónicos para interaccionar con el sistema nervioso
}

\section{Eduardo Fernández Jover}

Arbor CLXXVII, 698 (Febrero 2004), 311-335 pp.

Las enfermedades y accidentes que causan daños en el sistema nervioso suelen tener dramáticas consecuencias. Esto hace que en la actualidad se estén realizando grandes esfuerzos para el desarrollo de tratamientos capaces de restablecer las funciones perdidas. En este contexto, los recientes avances tecnológicos en el campo de la biomedicina, neurobiología e ingeniería, están permitiendo desarrollar y fabricar neuroprótesis que pueden llegar a proporcionar nuevas vías de tratamiento para la recuperación parcial de algunas de estas patologías. El propósito de este trabajo es presentar algunos ejemplos de sistemas de neuroprótesis en diferentes fases de evolución y discutir sus logros y sus limitaciones. Para ello hemos seleccionado algunas aplicaciones motoras y sensoriales que empiezan a estar ya disponibles y están proporcionando un gran ímpetu para el desarrollo de estas tecnologías. El futuro es esperanzador, sin embargo es necesario avanzar poco a poco y no crear falsas esperanzas que podrian afectar de forma negativa la credibilidad de estas investigaciones.

\section{Historia de la estimulación del sistema nervioso y su importancia para el desarrollo de futuras neuroprótesis}

Hoy en día, no sólo estamos empezando a comprender mejor la estructura y el funcionamiento del cerebro, sino que nuevas técnicas de ob- 
servación están aportando los medios necesarios para vincular directamente la dinámica molecular de las células nerviosas con representaciones de actos perceptivos y motores en el encéfalo, y para relacionar estos mecanismos internos con la conducta observable. Con esta capacidad para enlazar la biología molecular, las exploraciones funcionales y los procesos cognitivos, se están abriendo nuevos caminos para comprender el pensamiento y el comportamiento humano que además están permitiendo avanzar en el diagnóstico y tratamiento de muchas enfermedades. Así por ejemplo se están investigando nuevos tratamientos para las patologías degenerativas y existen ya dispositivos electrónicos que permiten restituir la audición en algunos pacientes sordos, la función vesical en pacientes con patología de la medula espinal, algunas funciones motoras en pacientes parapléjicos y tetrapléjicos, así como controlar el dolor crónico o los temblores de la enfermedad de Parkinson. Los principios biológicos básicos en los que se basan estos dispositivos son conocidos desde hace más de 150 años, por lo que nos podríamos preguntar: ¿porqué se ha tardado tanto tiempo en desarrollar dispositivos que puedan llegar a ser útiles para el tratamiento de patologías motoras y sensoriales?

Una forma de responder, al menos parcialmente, a esta pregunta es realizar un breve repaso de los acontecimientos históricos que han permitido estos importantes avances. Hace dos mil seiscientos años, mientras el oráculo de Delfos todavía vaticinaba el destino del hombre, Hipócrates $(s . V a . C)$ escribía:

«El hombre debería saber que, desde el cerebro, y exclusivamente desde el cerebro, surgen nuestros placeres, dichas, risas y bromas además de nuestras penas, dolores, tristezas y lágrimas. Mediante el cerebro pensamos, vemos y distinguimos lo feo de lo bello, lo malo de lo bueno, lo agradable de lo desagradable.... También nos hace delirantes o locos y nos infunde miedo o pavor, sea de noche o de día, así como los sueños y los delirios indeseables, las preocupaciones que no tienen razón de ser, la ignorancia de las circunstancias presentes, el desasosiego y la torpeza».

Sin embargo Aristóteles con su gran influencia, consiguió convencer a sus contemporáneos que era el corazón y no el cerebro el órgano encargado de las facultades mentales. Esta idea persistió hasta que Galeno (129200) aplicando la lógica aristotélica que aceptaba la experiencia sensorial como fuente del conocimiento científico, estableció una doctrina de la función cerebral que se mantuvo prácticamente sin cambios durante más de mil cuatrocientos años. Aparte de aceptar la refrigeración sanguínea en el 
cerebro y los pulmones, Galeno indicó que la sangre arterial se filtraba en la rete mirabile del cerebro y se convertía en pneuma psíquico que se acumulaba en el tercer ventrículo, desde donde pasaba a la médula espinal y a los nervios periféricos, para animar a las partes así inervadas. Esta idea persiste hasta la edad moderna. Así por ejemplo René Descartes (15961650), concibe el pneuma psíquico como un fluido, de una naturaleza peculiar aunque seguía siendo un fluido y por tanto susceptible de ser estudiado y comprendido aplicando las leyes que gobiernan los fluidos. Aplicando este concepto, elaboró una teoría del funcionamiento del sistema nervioso en la que este era concebido como una maquina, con el cerebro en la parte central y una serie de tubos (o nervios) que partiendo desde el cerebro llegaban a todas las partes del organismo. Una pequeña glándula, la glándula pineal, situada en el techo del tercer ventrículo constituía el reservorio principal. Las dilataciones ventriculares actuaban como un sistema adicional de reserva y permitían que el pneuma psíquico fluyera desde el cerebro y a través de los nervios (que eran tubos finísimos) para permitir los movimientos del cuerpo. En los años posteriores, este concepto empezó a experimentar una serie de transformaciones y cambios. Giovanni Borelli (1608-1679), contemporáneo de Descartes, sugirió la idea de la fermentación del fluido nervioso dentro de los músculos para explicar la contracción muscular. El fisiólogo Albrecht von Haller (1703-1777) desarrolló el concepto de irritabilidad del tejido nervioso que incluía la contractilidad innata de este y se empezó a considerar la posibilidad de que la electricidad pudiera ser el agente transmisor de la conducción nerviosa.

Aunque los fenómenos eléctricos y algunos de los efectos de la electricidad sobre los seres humanos eran conocidos desde la antigüedad, no fue hasta el siglo dieciocho cuando los investigadores empezaron a pensar en una posible conexión entre la electricidad y el funcionamiento del sistema nervioso. El abad Jean-Antoine Nollet (1700-1770), Benjamín Franklin (1706-1790) y otros investigadores demostraron que la energía procedente de las maquinas eléctricas de fricción era capaz de provocar contracciones musculares, incluyendo en algunos casos músculos que estaban paralizados. Este hecho indujo a pensar en la electricidad como una posible alternativa terapéutica para los enfermos con parálisis. Sin embargo esta observación no significaba necesariamente que los nervios y los músculos pudieran producir electricidad ni que utilizaran esta forma de energía para su funcionamiento.

La posibilidad de que la electricidad pudiera ser el agente transmisor de la conducción nerviosa había sido sugerida por Alexander Monro (1697-1762) y por el clérigo ingles Stephen Hales (1677-1761) que fue el primero en demostrar que la integridad de la medula espinal era esen- 
cial para los movimientos reflejos. En 1791 Luigi Galvani un médico y profesor italiano en Bologna, publicó un libro, De Viribus Electricitatis in Motu Musculari Commentarius, que ha representado una de las contribuciones mas importantes en la historia del funcionamiento del sistema nervioso (Figura 1). En la década de 1780-1790 se encontraba estudiando el problema de la «irritabilidad animal» y se preguntaba si los nervios eran conductores de un «fluido nervioso» análogo o igual al eléctrico. Para ello utilizaba una preparación neuro-muscular de rana ya que estos animales eran particularmente susceptibles de ser «irritados» por medio de inducción electrostática. Por casualidad observó que cuando encendía un generador electrostático, saltaban chispas de algunas de sus partes. En ese momento uno de sus ayudantes que estaba al otro extremo del laboratorio tocó con un bisturí de metal el nervio crural de una de las preparaciones de rana y se produjo una enérgica contracción muscular. Cuando el músculo fue conectado directamente a la máquina con un alambre, Galvani encontró que se contraía simultáneamente con la producción de electricidad. Entonces puso la máquina y la rana en cuartos diferentes y conectando con un alambre obtuvo los mismos resultados, aunque notó que conforme el alambre era más largo las contracciones eran más débiles. En cierto momento durante el curso de sus investigaciones, juntó de manera accidental, los alambres y se dio cuenta de que la pata también se contraía aunque ninguno de los extremos estaba conectado a ninguna fuente de carga eléctrica. Galvani no entendía claramente los mecanismos físicos y químicos que daban lugar a estas sorprendentes contracciones, pero suponía que el cerebro era la fuente de esta «electricidad animal» y que esta era posteriormente distribuida por todo el sistema nervioso a través de los nervios. En este sentido propuso que la rana y todos los otros seres vivos poseían una electricidad inherente y escribió:

«naturalmente, éste resultado produce no poca sorpresa en nosotros y empieza a dar origen a la sospecha de que la electricidad es inherente al animal mismo».

Estos experimentos fueron puestos en duda por un físico italiano, Alesandro Volta (1745-1827), quien criticó severamente los resultados de Galvani y demostró que los diferentes metales usados en los experimentos de Galvani habían sido la fuente de electricidad para estimular las contracciones musculares de la rana. Volta se dio cuenta de que para lograr el efecto descubierto por Galvani se necesitaba cobre, hierro y el líquido del tejido muscular. Hizo una serie de experimentos muy cuidadosos, utilizando alambres de diferentes materiales; y descubrió que si usaba estaño y cobre lograba una corriente relativamente fuerte, mientras que si usaba 
Nuevos dispositivos electrónicos para interaccionar...

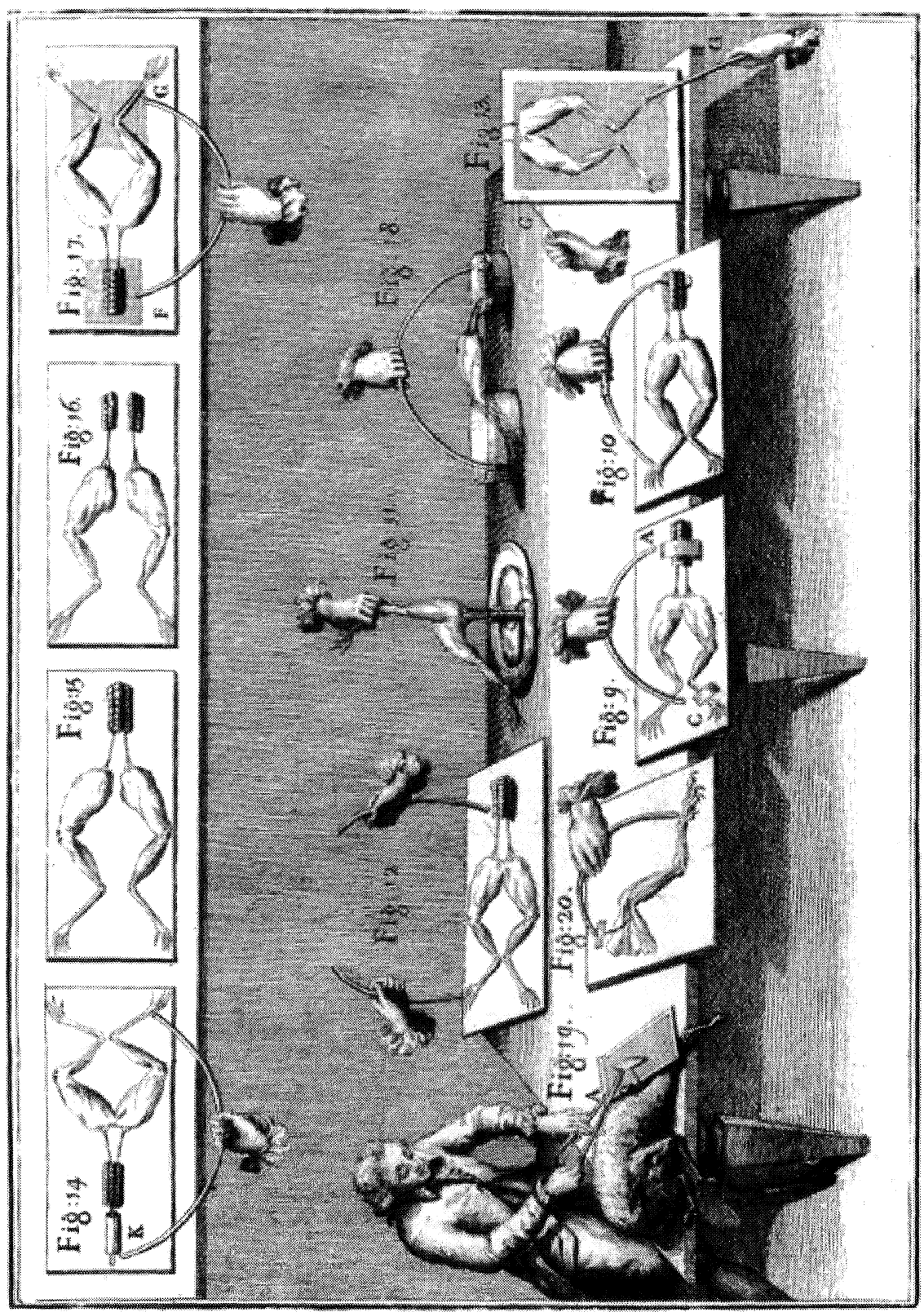

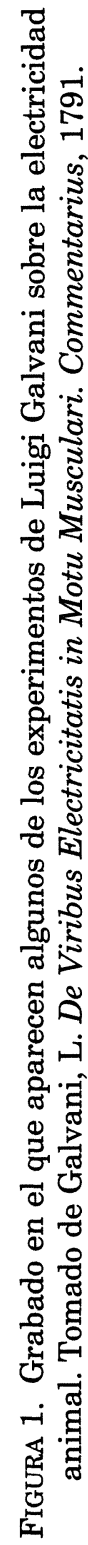


hierro y plata el efecto era poco intenso. De esta manera llegó a la conclusión de que el efecto descubierto por Galvani no tenía nada que ver con la denominada «electricidad animal» sino que se debía a una acción química entre el líquido, llamado electrolito, y los dos metales. Es así como Volta construyó lo que posteriormente se llamó una pila voltaica, que fue el primer dispositivo electroquímico que sirvió como fuente de electricidad. En cualquier caso y aunque Galvani estaba equivocado en la interpretación de sus resultados, demostró la importancia de la electricidad en la contracción muscular y sus trabajos abrieron el camino para el estudio de los mecanismos de la generación y propagación de las señales eléctricas dentro del sistema nervioso y para el desarrollo de las modernas neuroprótesis.

En 1843, Emil Du Bois-Reymond rompió completamente con la tradición vitalista y consiguió explicar los fenómenos bioeléctricos en términos de principios físicos y químicos. Demostró que el flujo de corriente eléctrica intervenía no solo en la contracción muscular sino también en la conducción nerviosa, y que el hecho de que la transmisión de la información entre el nervio y el músculo sea mediada por el flujo de corriente no es sino una pequeña extensión de este concepto. Sin embargo, hasta la mitad del siglo XX, con los experimentos de Hodgkin y Huxley no fue posible aclarar completamente la naturaleza de la denominada «electricidad animal» y las bases biológicas de la transmisión del impulso nervioso. La solución a este viejo problema se basó en dos hechos: el descubrimiento y la utilización de una preparación biológica apropiada, el axón gigante del calamar, y el desarrollo de aparatos de medida más sensibles. Con la preparación de axón gigante se hizo posible, por primera vez, la inserción de electrodos en el interior de una fibra nerviosa (antes sólo se podía registrar la actividad de los nervios y de los músculos por medio de electrodos aplicados exteriormente). Con esta técnica se pudo demostrar que las señales que utilizan la células nerviosas para transmitir su información consisten en corrientes eléctricas generadas entre el interior y el exterior de la membrana que las envuelve. Estas corrientes se forman por el flujo de iones a través de los compartimentos intracelular y extracelular y se deben principalmente a los movimientos de cargas transportadas por los iones sodio, potasio, calcio y cloro.

\section{Bases fisiológicas de la comunicación entre las células nerviosas y los dispositivos electrónicos}

Las células nerviosas o neuronas, son las piezas clave de la construcción del sistema nervioso y parte de la complejidad cerebral reside en la 
diversidad de estas células a las que Santiago Ramón y Cajal, padre de la moderna neurociencia, describió como:

"... las misteriosas mariposas del alma, cuyo batir de alas quién sabe si esclarecerá algún día el secreto de la actividad mental».

Las neuronas están compuestas básicamente por una membrana que delimita un compartimiento en cuyo interior se encuentra una solución acuosa de sales y proteínas. La membrana celular limita la difusión de iones y separa el interior celular del exterior, que en un sentido amplio tiene la misma composición iónica pero diferente concentración. Las neuronas envían información a otras neuronas generando potenciales de acción, que son señales eléctricas que se propagan a lo largo del único axón de la célula a modo de olas, y son convertidas en señales químicas a nivel de las sinapsis o contactos con otras células. Por tanto, el funcionamiento del sistema nervioso depende en gran medida del flujo de información en forma de corrientes eléctricas a través de elaborados circuitos neuronales. Sin embargo, el interior celular es peor conductor de electricidad que los hilos metálicos, porque el número de portadores de carga es más pequeño y su movilidad es menor. Estos factores limitan inevitablemente la cantidad de corriente que una fibra nerviosa puede conducir. La conducción de corriente a lo largo de los nervios está dificultada además por el hecho de que la membrana es un aislante imperfecto y, por consiguiente, permite cierto paso de sustancias entre el medio externo e interno. Además de estos defectos puramente eléctricos, las fibras nerviosas son pequeñas, lo que limita aún más la cantidad de corriente. Hodkin (1964) expuso un ejemplo muy gráfico de las consecuencias que pueden tener estos factores sobre la difusión de las señales eléctricas:

«Si un ingeniero eléctrico tuviera que examinar el sistema nervioso, lo primero que advertiría es que la transmisión de información a lo largo de las fibras nerviosas por medio de señales eléctricas es un tremendo problema. El diámetro de los axones varia entre 0.1 y $10 \mu \mathrm{m}$. El interior de la fibra nerviosa contiene iones y es bastante buen conductor de la electricidad. Sin embargo, la fibra es tan pequeña que su resistencia longitudinal resulta excesivamente elevada. Con un simple cálculo se verá que una fibra de $1 \mathrm{~mm}$ de diámetro y una resistividad de 100 ohmios por centímetro tendrá una resistencia por unidad de longitud de aproximadamente $10^{10}$ ohmios por centímetro. Esto significa que la resistencia eléctrica de un metro de fibra nerviosa de pequeño calibre es casi la misma que la de $10^{10}$ millas de hilo de cobre de calibre 22, que representa, más o menos, diez veces la distancia existente entre la Tierra y el planeta Saturno» 
Sin embargo el sistema nervioso consigue no sólo superar sino también aprovechar todos estos defectos, y también lo han hecho los investigadores que trabajan en el campo de las neuroprótesis.

Hasta donde conocemos, existen básicamente 2 tipos de sistemas físicos capaces de procesar información en tiempo real: a) los circuitos electrónicos en los que el flujo de información se realiza por electrones que circulan sobre conductores metálicos y b) los circuitos neuronales en los que la información se transmite a través de iones que se desplazan en un medio acuoso como es el medio intracelular. Las neuroprótesis no son más que dispositivos o sistemas en los que puede intercambiarse información entre estos 2 tipos de circuitos con el fin de intentar recuperar o restituir, al menos parcialmente, algunas de las funciones perdidas. Dicho así, puede dar la impresión de que estamos hablando de ciencia ficción, pero ciertamente no es el caso ya que muchos de estos dispositivos son ya una alternativa clínica real como por ejemplo los marcapasos que se utilizan desde hace más de 40 años para la estimulación eléctrica del tejido cardiaco, los implantes cocleares para pacientes con déficits auditivos, los estimuladores del nervio frenico para tratar algunos tipos de insuficiencia respiratoria, la estimulación del nervio vago en algunos tipos de epilepsia, la estimulación de la medula espinal para disminuir el dolor originado por trastornos como la retinopatía diabética periférica o la estimulación de centros cerebrales profundos en el caso de la enfermedad de Parkinson. Dos importantes líneas de investigación están surgiendo en este contexto. La primera nace a partir de la observación de que los procesos biofísicos implicados en la estimulación y registro de las células nerviosas son universales, y por tanto los avances tecnológicos en cualquiera de estas áreas tienen amplias repercusiones en muchas disciplinas clínicas. La segunda esta relacionada con la oportunidad que se presenta a los investigadores de realizar experimentos neurofisiológicos y psicológicos directamente en seres humanos.

\section{Neuroprótesis y patologías del sistema nervioso}

Las enfermedades y accidentes que causan daños en el sistema nervioso, suelen tener dramáticas consecuencias. Una de las principales razones es que la regeneración nerviosa y el restablecimiento de las conexiones sinápticas adecuadas no es posible en la mayor parte de los casos. Otra razón es que las neuronas muertas no pueden ser reemplazadas con nuevas neuronas. Ello ha dado lugar a grandes esfuerzos para el desarrollo de sistemas capaces de interaccionar con el sistema nervioso, y res- 
tablecer, al menos parcialmente las funciones perdidas. Este es un campo relativamente nuevo que esta avanzando de manera muy rápida gracias al trabajo en equipo de científicos de muchas disciplinas diferentes (medicina, biología, microelectrónica, bioingeniería, tecnologías de la información, etc). Cuando Benjamín Franklin definió al hombre como «un animal que fabrica herramientas», en esta definición estaba implícito el hecho de que el hombre se ha especializado siempre en la fabricación de sustitutos para las partes del cuerpo que no posee (por ejemplo cuchillos en lugar de dientes para desgarrar eficazmente la carne) pero no sospechaba que en la actualidad y gracias a los avances en los conocimientos médicos, científicos y de la ingeniería iba a ser posible empezar a plantearse la sustitución de elementos del organismo humano que han quedado dañados como consecuencia de procesos patológicos degenerativos o traumáticos.

La figura 2 presenta un resumen de algunos de los sistemas de neuroprótesis que se encuentran actualmente en fase de desarrollo. En general podemos distinguir sistemas capaces de reemplazar alguna de las funciones nerviosas que se han perdido como consecuencia de una enfermedad o lesión, o bien dispositivos que permiten aumentar o reemplazar cualquier defecto o alteración en la información que desde los órganos sensoriales periféricos debe llegar hasta el sistema nervioso central. En el primer caso las neuroprótesis actúan como intermediarios entre diferentes elementos del sistema nervioso y los sistemas musculares cuyo control se ha perdido. Un ejemplo de este tipo de neuroprótesis son los sistemas para recuperar parcialmente la movilidad de la mano y la extremidad superior en pacientes tetrapléjicos. En el segundo caso los dispositivos electrónicos permiten registrar y procesar las señales externas y transmitir esta información a los centros de procesamiento cerebral. Algunos ejemplos son los implantes cocleares para recuperar la percepción auditiva o las neuroprótesis para restituir la visión.

El propósito de este trabajo es presentar algunos ejemplos de sistemas de neuroprótesis en diferentes fases de evolución y discutir sus logros y sus limitaciones. Para ello hemos seleccionado algunas aplicaciones motoras y sensoriales que empiezan a estar ya disponibles y están proporcionando un gran ímpetu para el desarrollo de estas tecnologías. En el año 1998, la $\mathrm{FDA}^{1}$ americana (agencia que se encarga de aprobar los medicamentos y los nuevos dispositivos terapéuticos) aprobó la utilización comercial de las primeras neuroprótesis para el control de la función vesical, y el primer amplificador auditivo integrado (TICA) fue implantado en Europa. En el año 2000, la FDA aprobó las primeras prótesis auditivas para el tratamiento de patologías auditivas del oído medio y un 


\section{Eduardo Fernández Jover}

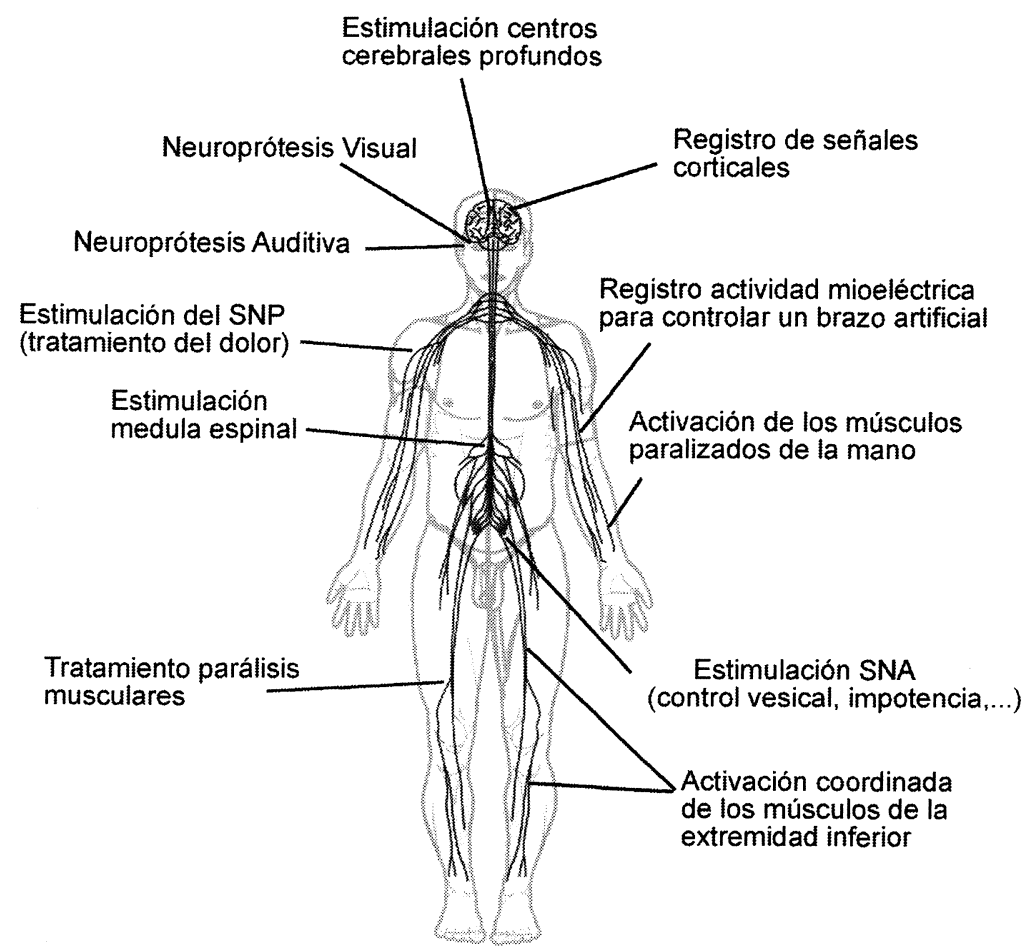

FIgURA 2. Algunos sistemas de neuroprótesis que se encuentran actualmente en fase de desarrollo.

nuevo dispositivo para el control de la vejiga urinaria en humanos (Interstim) y en Europa, se aprobó la utilización clínica de la primera neuroprótesis auditiva completamente desarrollada en Alemania (Implex AG Hearing Technology). Al año siguiente la FDA aprobó el primer sistema protésico para recuperar la funcionalidad de la mano en pacientes amputados (Handmaster System) y paralelamente un grupo Europeo liderado por el Prof. Pierre Rabischong (Universidad de Montpellier) consiguió devolver la capacidad de mover las piernas e incluso ponerse en pie a varios pacientes parapléjicos. En los últimos 2-3 años, algunos grupos de investigación (Donoghue et al., 2002; Nicolelis et al., 2003) están explorando la posibilidad de registrar, mediante electrodos intracorticales, la actividad de la corteza motora cerebral en enfermos con parálisis severas y utilizar esta información para controlar directamente dispositivos externos, mientras que otros están dirigiendo sus esfuerzos a utilizar una tecnología similar para la restauración de la visión y otras capacidades sensoriales perdidas (Badi et al., 2003; Branner et al., 2004; Fernández 
Nuevos dispositivos electrónicos para interaccionar...

et al., 2004; Hillman et al., 2003). Todos estos dispositivos podrían representar un gran avance en la mejora de la calidad de vida de las personas afectadas por estas patologías y abren todo un mundo de posibilidades para los enfermos que permanecen incomunicados o incapacitados a causa de lesiones de su sistema nervioso, deficiencias sensoriales o ausencia de miembros. En este contexto el importante desarrollo de las ciencias biomédicas y la bioingeniería en los últimos años, está conduciendo a un rápido aumento de nuestros conocimientos y permitiendo desarrollar dispositivos que eran impensables hace pocos años.

\section{Implantes para restituir la audición}

La colocación de implantes electrónicos en la cóclea, componente esencial del oído interno, representa uno de los ejemplos más exitosos de los implantes neuroprotésicos. El primer implante coclear fue desarrollado por Djourno y Eyries en 1957. En la década 1970-1979 y gracias a los avances en microelectrónica, se perfeccionaron considerablemente estos dispositivos electrónicos y comenzaron las primeros ensayos clínicos en humanos en Estados Unidos. Unos años mas tarde se iniciaron los primeros ensayos clínicos en Japón (1983) y poco a poco, esta tecnología ha ido consiguiendo una amplia aceptación pública y comercial, presentándose actualmente como un claro ejemplo de lo que puede llegar a ser posible en este campo.

En el oído sano las vibraciones sonoras son transmitidas y concentradas hacía el tímpano o membrana timpánica. Las ondas sonoras que entran en contacto con el tímpano son convertidas en vibraciones mecánicas y amplificadas gracias a un sistema de huesecillos (martillo, yunque y estribo) que las transmiten a la cavidad del oído interno. Posteriormente el líquido situado dentro del oído interno vibra y estas vibraciones estimulan las aproximadamente 30.000 células sensitivas que se encuentran dentro de una estructura de forma espiral denominada órgano de Corti. En cada punto de la espiral, la membrana está sintonizada para vibrar a una determinada frecuencia de las ondas sonoras que llegan al oído, de manera que en general sólo vibra un punto específico para cada frecuencia sonora. Esto produce la activación de las células sensitivas adecuadas y la producción de impulsos eléctricos que se envían a través de los nervios auditivos al tronco cerebral y a la corteza auditiva del cerebro.

Las células sensitivas, denominadas células ciliadas, pueden disminuir en número o verse alterado su funcionamiento a consecuencia de enfermedades congénitas o adquiridas. Algo similar puede ocurrir como 


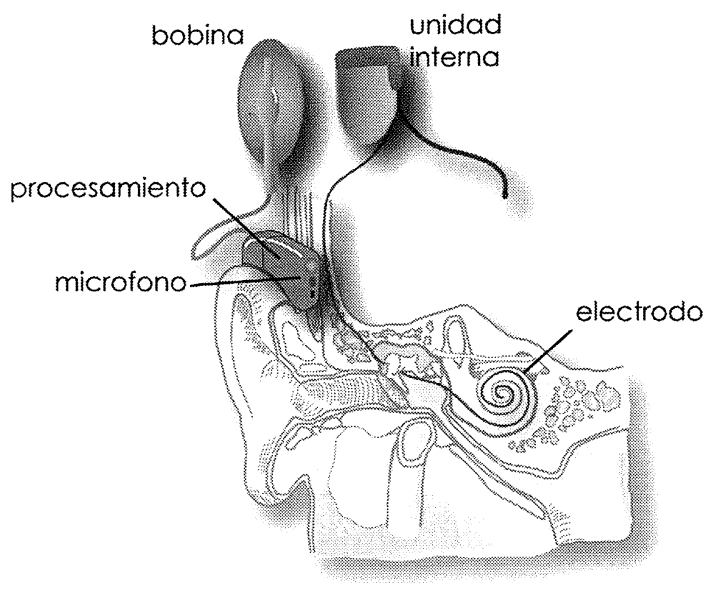

Figura 3. Esquema de los elementos básicos de un implante coclear. El sonido es captado por un micrófono, que convierte las ondas sonoras en estímulos eléctricos que son utilizados para estimular directamente el nervio auditivo. La información desde la unidad de procesamiento externa se transmite a la unidad interna, mediante un sistema inalámbrico.

consecuencia de daños traumáticos o asociado a los procesos del envejecimiento. En cualquier caso, la consecuencia inmediata de la alteración de las células sensitivas del órgano de Corti es la aparición de sordera o incapacidad para percibir estímulos sonoros. En estos casos las ayudas auditivas externas, que sólo amplifican la intensidad del sonido, no permiten recuperar la audición. Cuando todavía se mantiene la integridad del nervio auditivo es posible estimularlo directamente y de manera selectiva mediante electrodos implantados en la parte del oído interno donde reside el órgano de Corti, es decir en la cóclea, y eso es exactamente lo que hacen los implantes cocleares. Estos implantes sustituyen a las estructuras del oído dañadas y son utilizados con éxito en miles de pacientes. Un esquema general se presenta en la Figura 3.

Cuando existe alteración en los propios nervios auditivos (a consecuencia de procesos degenerativos, traumáticos, tumorales, etc.) los implantes cocleares no representan una elección terapéutica útil. En estos casos, es posible plantearse la estimulación directa del siguiente paso en la vía auditiva, es decir los centros cerebrales que participan en el procesamiento de la información auditiva. Esta alternativa es posible y puede hacerse utilizando electrodos que se implantan en el tronco del encéfalo, a nivel de los núcleos cocleares. Cuando se opta por esta opción, son más efectivos los electrodos que penetran dentro las estructuras profun- 
Nuevos dispositivos electrónicos para interaccionar...

das del tejido nervioso, que los que se colocan en la superficie del mismo ya que permiten estimular las células adyacentes a los microelectrodos con un mayor rango dinámico y menor cantidad de corriente. Un avance importante en este campo ha sido la incorporación, durante la implantación quirúrgica, del registro de la actividad eléctrica de las poblaciones de células nerviosas que se encuentran en la proximidad de los electrodos para localizar el lugar idóneo de implantación. Estas técnicas de registro y estimulación también se están utilizando actualmente para guiar la colocación de los electrodos de estimulación crónica en el tratamiento de la enfermedad de Parkinson y están poniendo de manifiesto la gran relevancia que tiene la posibilidad de una comunicación bidireccional de información en el desarrollo de estas tecnologías.

El éxito de los implantes para restituir la audición puede variar de unos pacientes a otros debido a la heterogeneidad de los trastornos y al diferente grado de destrucción del órgano de Corti. La colocación precoz de este tipo de implantes permite a muchos niños sordos adquirir unas excelentes competencias lingüísticas, sin embargo en los adultos, los resultados suelen ser mas decepcionantes. Estos resultados parecen estar en relación con las modificaciones plásticas que se producen en el cerebro como consecuencia de la deprivación auditiva y los fenómenos de reorganización funcional de la corteza cerebral. De todas formas hay que tener en cuenta que cuando la FDA aprobó la utilización de los primeros implantes auditivos, los pacientes candidatos a este tipo de tratamiento tenían que padecer una sordera total. En el año 1995 se revisaron estos criterios para incluir a pacientes con una discapacidad auditiva importante que no consiguieran mejorar su audición en más de un $30 \%$ con ayudas externas. Este cambio refleja los importantes avances en estas tecnologías, que en gran medida están vinculados a los avances en microelectrónica, neurociencias y bioingeniería, así como sus posibilidades futuras.

\section{Estimulación Neuromuscular}

En muchos tipos de parálisis, incluyendo aquellas causadas por infartos cerebrales y lesiones de la medula espinal, la parte más periférica del sistema neuromuscular se mantiene intacta. Desde los experimentos de Galvani se conoce que la estimulación eléctrica es capaz de inducir la contracción de los músculos, por lo que el principal problema clínico en este campo es el control coordinado de diferentes masas musculares para generar movimientos. Para ello es importante conocer que información se 
transmite desde nuestros «centros de control» y este es uno de los grandes retos de la neurobiología actual.

El objetivo de algunos de estos dispositivos, es estimular eléctricamente el sistema neuromuscular en pacientes con lesiones neurológicas para recuperar su funcionalidad. En este caso utilizan la estimulación eléctrica para generar ejercicios musculares pasivos, que probablemente desencadenan la liberación de factores tróficos y ejercen otros efectos beneficiosos que permiten mejorar los tratamientos habituales de rehabilitación.

Actualmente existen también estimuladores neuromusculares que permiten controlar los músculos del brazo y la mano en pacientes que han sufrido daños en el sistema nervioso central como consecuencia, por ejemplo, de infartos cerebrales. Una de las líneas de investigación más interesantes dentro de este campo, se basa en el microestimulador BION, que mide aproximadamente $2,5 \mathrm{~mm}$ de diámetro y unos $16,5 \mathrm{~mm}$ de largo y puede ser fácilmente implantado en los músculos utilizando una aguja especial (Loeb y Richmond, 2001). Cada estimulador BION puede controlarse con independencia, con un máximo de 255 dispositivos. Este sistema es menos invasivo que otras generaciones anteriores de implantes neurológicos, y resuelve muchos de los inconvenientes cosméticos y de utilización asociados a los sistemas de estimulación externos.

Este es un campo que esta en continuo desarrollo. Por ejemplo se están consiguiendo unos resultados espectaculares en el desarrollo de sistemas de estimulación eléctrica funcional capaces de devolver la capacidad de mover las piernas a pacientes parapléjicos, y sistemas que permitan recuperar el movimiento de los brazos a pacientes tetrapléjicos. A partir de las medidas de los movimientos, las fuerzas generadas por la estimulación, los efectos de la actividad de cada uno de los músculos estimulados aisladamente y en coordinación con otros músculos y la capacidad de los pacientes de controlar su movimiento, se están desarrollando sistemas muy sofisticados de control que permiten que algunos sujetos parapléjicos, que todavía conservan su masa muscular en buen estado, sean capaces de ponerse en pie e incluso andar. En cualquier caso todavía se necesita más investigación para conocer que información se transmite desde nuestros «centros de control» para, por ejemplo, ordenar los movimientos necesarios para coger un objeto. También es necesario conocer que señales se producen en las articulaciones, en los músculos y en las yemas de los dedos, para controlar el movimiento e informar al cerebro de lo que está sucediendo. En este sentido se ha conseguido ya decodificar algunas de las señales emitidas por el cerebro para controlar la motricidad de los miembros y se están perfeccionando los electrodos y los 
Nuevos dispositivos electrónicos para interaccionar...

sistemas de estimulación que en el futuro podrían integrar dispositivos capaces de liberar factores favorecedores de la regeneración, factores neurotróficos e incluso terapias génicas.

\section{Neuroprótesis visuales}

La investigación de la búsqueda de alternativas que ayuden a personas ciegas o con baja visión residual, a mejorar su movilidad e incluso de una forma más ambiciosa a percibir el entorno que les rodea y orientarse en él, puede hacerse desde distintos puntos de vista. Las degeneraciones retinianas como la retinosis pigmentosa y la degeneración macular, inducen alteraciones visuales que a menudo ocasionan cegueras intratables, por lo que varios laboratorios se encuentran actualmente trabajando en el desarrollo de prótesis visuales diseñadas para interaccionar con las porciones no dañadas de la retina o el nervio óptico (Chow et al., 2001; Eckmiller, 1997; Lakhanpal et al., 2003; Rizzo et al., 2003; Zrenner, 2002; Zrenner et al., 1999; Delbeke et al., 2002, 2003; Veraart et al., 2003). Los resultados preliminares con este tipo de dispositivos son esperanzadores aunque de momento sólo se han utilizado un número muy reducido de microelectrodos. En cualquier caso ya se han iniciado en Estados Unidos los primeros ensayos clínicos en pacientes con retinosis pigmentosa y degeneración macular.

Sin embargo, en muchos casos existe una mayor o menor afectación de todas las células de la retina, por lo que la utilidad de las neuroprótesis visuales a nivel de la retina o nervio óptico puede verse seriamente comprometida. Además estos tipos de neuroprótesis no permiten el tratamiento de cegueras cuya patología afecte a las células ganglionares de la retina, al nervio óptico o las vías visuales extraoculares. Una posible alternativa, en la que nosotros estamos trabajando en colaboración con varios grupos europeos y el profesor Normann de la Universidad de Utah, USA, es la implantación de electrodos microelectrodos a nivel de la parte del cerebro que se encarga de procesar la información visual, la corteza occipital. El principal objetivo de esta aproximación terapéutica, es el desarrollo de una neuroprótesis cortical que pueda ayudar a personas ciegas, o con baja visión residual, a mejorar su movilidad e incluso de una forma más ambiciosa a percibir el entorno que les rodea y orientarse en él.

Esta línea de trabajo se basa en el hallazgo de que la estimulación eléctrica de la corteza occipital desencadena la percepción subjetiva de destellos de luz denominados técnicamente fosfenos. El primer trabajo sobre la aparición de fosfenos tras la estimulación eléctrica del cortex visual se debe a Lowënstein y Borchart en 1918, pero fueron los estudios 
del neurocirujano canadiense Wilder Penfield en la década de 1950, los que confirmaron estos hallazgos. Más tarde Brindley y Lewin en la Universidad de Cambridge y el grupo de Dobelle en la Universidad de Utah hicieron prolongadas observaciones sobre los fosfenos y los estímulos eléctricos que los desencadenaban, sentando las bases de una neuroprótesis visual a nivel cortical. En este sentido hay que destacar los trabajos del grupo de Dobelle en los que, sujetos que habían permanecido ciegos durante largo tiempo, fueron capaces de leer caracteres Braille utilizando un sistema de electrodos incluso más rápido de lo que lo hacían a través del tacto. Los resultados de estos estudios han puesto sin embargo de manifiesto que una neuroprótesis basada en la estimulación cortical mediante electrodos superficiales, tal como la que ha sido presentada recientemente por el Dr. Dobelle (Dobelle, 2000), puede tener una utilidad limitada, debido fundamentalmente a factores tales como la gran cantidad de corriente necesaria para producir los fosfenos, interacciones entre electrodos adyacentes, la producción ocasional de episodios dolorosos debidos probablemente a irritación meníngea, y a la posibilidad de desencadenar crisis epilépticas.

Una aproximación más eficaz, que permite la activación de neuronas con un mayor grado de especificidad espacial y menores niveles de corriente, es la utilización de electrodos intracorticales (Maynard, 2001; Normann et al., 1999; Rousche y Normann, 1999). En este sentido, Ed Schmidt y sus colaboradores (1996) implantaron 38 microelectrodos intracorticales en el cortex visual derecho de una mujer de 42 años que estaba ciega desde hacía 22 años. 34 de estos electrodos fueron capaces de producir fosfenos durante un periodo de 4 meses con niveles de corriente inferiores a $25 \mu \mathrm{A}$. Desafortunadamente estos electrodos no fueron diseñados para su uso crónico por lo que algunas de sus conexiones se rompieron en los primeros días del experimento. En cualquier caso, todas estas investigaciones sugieren que un sistema de electrodos múltiples, insertado en los lugares apropiados del sistema visual, es capaz de inducir la percepción subjetiva de fosfenos, y podría ser utilizado para proporcionar una visión limitada pero útil a un gran número de personas ciegas . Los principales fundamentos fisiológicos que apoyan esta aproximación experimental son:

- La mayor parte de las cegueras son de causa retiniana y dejan los centros visuales superiores (corteza cerebral) intactos (Normann et al. 1996; Fernández et al., 2004).

- La estimulación eléctrica de neuronas de la vía visual produce la percepción subjetiva de fosfenos (Brindley y Lewin, 1968, Brindley 
Nuevos dispositivos electrónicos para interaccionar...

et al., 1972, Dobelle y Mladejovsky 1974, Dobelle et al., 1974, 1976, Schmidt et al., 1996).

- Las vías visuales se organizan de forma sistemática, siguiendo una alta organización retinotópica. Desde los trabajos pioneros de Brodmann, se ha avanzado mucho en el conocimiento de la organización de las vías visuales y hoy en día sabemos que la información de las células ganglionares procedentes de las mitades ipsilateral y contralateral de la retina terminan en diferentes zonas de la corteza visual primaria. Además la entrada de información a la corteza visual es razonablemente coherente y la estimulación simultánea de múltiples fosfenos puede inducir la percepción de patrones complejos.

- Experiencia clínica con otros tipos de dispositivos electrónicos diseñados para interaccionar con el tejido neural (marcapasos, prótesis auditivas, estimuladores para tratamiento del dolor crónico, etc). Este tipo de neuroprótesis son ya habituales en clínica humana y se posee amplia experiencia en su fabricación y desarrollo.

- Plasticidad del sistema nervioso en general y el sistema visual en particular. Por ejemplo son ya clásicos los trabajos en los que se coloca a sujetos voluntarios unas lentes que hacen que todo lo vean distorsionado o al revés (Bedford, 1999; Harris, 1965; Sekiyama et al., 2000). Al principio estos sujetos tienen mareos y problemas para mantener la posición. Sin embargo después de algunos días son capaces de moverse lentamente en un entorno familiar, y en unas semanas aprenden a realizar tareas motoras complejas como dibujar, escribir o incluso montar en bicicleta y jugar al tenis. Estos resultados sólo son posibles si el sistema visual de estos sujetos adultos es capaz de remodelarse o adaptarse a estas nuevas condiciones. Así, el sistema visual es una red neural con una gran capacidad de adaptación, por lo que al igual que sucede con los implantes cocleares cabe esperar que con el entrenamiento adecuado, los sujetos portadores de estas neuroprótesis aprenderán a asociar los patrones de fosfenos con el mundo físico exterior (inmediatamente después de la implantación, los fosfenos desencadenaran una pobre percepción, sin embargo con el paso del tiempo y el entrenamiento apropiado, los sujetos aprenderán a reconocer e interpretar cada vez mejor estos patrones).

Los principales requisitos del sistema son en general bien conocidos. $\mathrm{Al}$ igual que sucede con cualquier otra neuroprótesis diseñada para interaccionar de forma efectiva con el tejido nervioso, el sistema debe ser capaz de: 
1. Registrar el mismo tipo de información que normalmente utiliza el sistema. En este caso, al tratarse de una prótesis visual, deben ser capaces de registrar información visual.

2. Procesar esta información de la manera más «fisiológica» posible.

3. Transmitir y comunicar esta información, una vez procesada, a los sitios adecuados dentro del sistema nervioso.

Además debe estar optimizada para el tratamiento de una determinada patología o para la recuperación de una determinada función, ser adaptable a cada paciente, biocompatible, y poseer un tamaño adecuado y un bajo consumo. Un esquema del sistema completo se presenta en la Figura 4. El campo visual situado enfrente del sujeto será codificado por una retina artificial bioinspirada, capaz de captar y procesar la información visual de una forma similar a como lo hace la retina humana. Las señales procedentes de este dispositivo serán procesadas externamente y transformadas en trenes de impulsos eléctricos optimizados para estimular las neuronas de la corteza visual a través de microelectrodos intracorticales. La comunicación con los electrodos intracorticales será inalámbrica, utili-

A

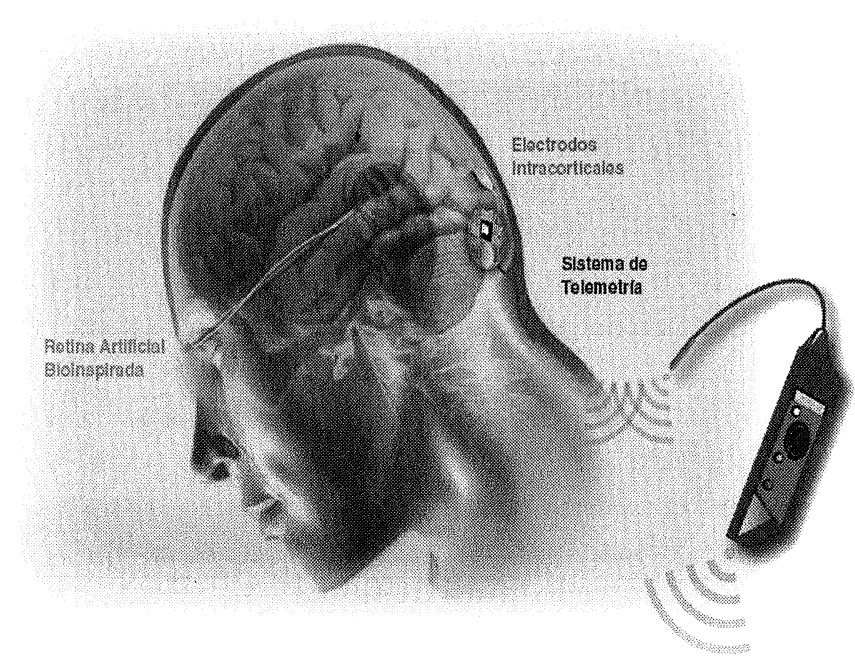

B

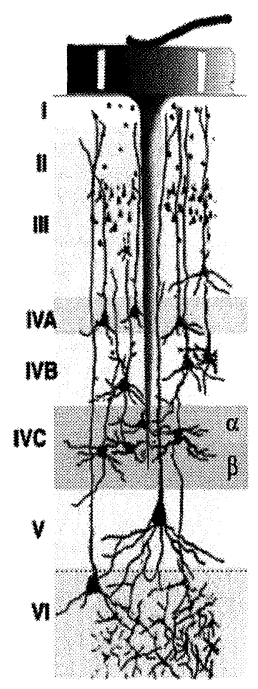

Figura 4. Dibujo esquemático de una neuroprótesis visual a nivel cortical. A, El campo visual situado enfrente del sujeto será codificado por un pequeño dispositivo electrónico bioinspirado (retina artificial) situado a nivel de unas gafas convencionales. Las señales procedentes de esta retina artificial serán procesadas externamente y transformadas en trenes de impusoso eléctricos optimizados para estimular las neuronas de la corteza visual primaria a través de una matriz de microelectrodos intracorticales. B, Implantación en la corteza cerebral. 
Nuevos dispositivos electrónicos para interaccionar...

zando sistemas de telemetría que permitan disminuir los riesgos de infección asociados con las comunicaciones percutáneas estándar.

Para que esta aproximación pueda ser realmente eficaz, no es necesario transmitir una imagen con una alta resolución y de todo el campo visual, sino transmitir una información útil (para tareas tales como leer, orientación y movilidad) a los sitios adecuados dentro del sistema visual. Para ello es imprescindible conocer como se codifica la información en la retina y como se transmite esta información a la corteza visual, por lo que este es una de nuestras principales líneas de investigación. Además es necesario que el organismo acepte bien este tipo de implantes. En este sentido la matriz de microelectrodos que estamos utilizando (Figura 5) ha sido utilizada con éxito en experimentos agudos y crónicos de estimulación y registro, y existen estudios que avalan su seguridad, biocompatibilidad y estabilidad (Branner et al., 2004; Fernández et al., 2004; Maynard et al., 2000; Normann et al., 1999, 2001; Warren et al., 2001). Por otro lado, es importante que la inyección de corriente a nivel cerebral sea efectiva y segura. Nuestros resultados preliminares en animales de experimentación son muy alentadores y nos están permitiendo conocer los parámetros mas adecuados para conseguir una estimulación eficaz y no lesiva (Fernández et al., 2004).

Otro prerrequisito importante para la aplicación clínica de este tipo de neuroprótesis, además de que el organismo la acepte y no provoque daños, es que la corteza visual de los candidatos a esta alternativa terapéutica, sea todavía capaz de procesar información visual. Existen estudios que han puesto de manifiesto la existencia de una serie de adap-

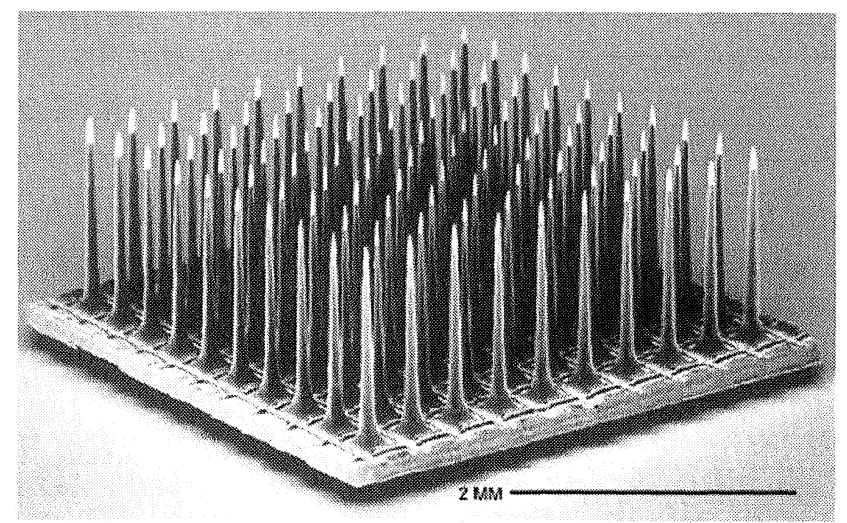

FIGURA 5. Imagen de microscopía electrónica de barrido de una matriz e electrodos intracorticales (cedida amablemente por el Dr. R.A. Normann de la Universidad de Utah) 
taciones a la perdida de visión, que se caracterizan fundamentalmente por un reclutamiento de la parte del cerebro que habitualmente se encarga de procesar información visual (corteza occipital) para el procesamiento de otras modalidades sensoriales como la somatoestésica (sensibilidad, tacto) y auditiva (Cohaen et al., 1997; Pascual-Leone et al., 1999). Esta plasticidad cortical transmodal, se asocia en general con una mejor adaptación de los individuos ciegos a su discapacidad sensorial, aunque no sabemos si se produce por igual en todos los sujetos ciegos. Nuestros datos sugieren que los sujetos con importantes déficits visuales, pero no absolutamente ciegos, junto con los que se han quedado ciegos tardíamente (a consecuencia de procesos degenerativos, accidentes,....) tienen más posibilidades de que su corteza visual pueda responder todavía a estímulos visuales. Estos pacientes no acaban de adaptarse bien a la perdida de la visión, y para ellos una neuroprótesis cortical podría ser una buena alternativa terapéutica. En este contexto es importante desarrollar tecnologías y protocolos que puedan ser utilizadas para el estudio y evaluación pre-quirúrgica de los posibles candidatos a una neuroprótesis cortical (Fernández et al., 2002).

\section{El futuro: promesas y problemas potenciales}

Decía José Ortega y Gasset en su Meditación de la Técnica (1939)

«Uno de los temas que en los próximos años se va a debatir con mayor brío es el del sentido, ventajas, daños e inconvenientes de la técnica. Siempre he considerado que la misión del escritor es prever con holgada anticipación lo que va a ser problema, años más tarde, para sus lectores y proporcionarles a tiempo, es decir, antes de que el debate surja, idas claras sobre la cuestión, de modo que entren en el fragor de la contienda con el ánimo sereno de quien, en principio, ya la tiene resulta».

Estas reflexiones son hoy mas ciertas que nunca. Los recientes avances tecnológicos en el campo de la neurobiología, neurología, neurocirugía, biomedicina, e ingeniería, están permitiendo desarrollar y fabricar neuroprótesis que puedan llegar a ser consideradas como una alternativa clínica real para la recuperación parcial de algunas enfermedades y degeneraciones del sistema nervioso (Heiduschka y Thanos, 1998). En este contexto, el viejo sueño humano de desarrollar dispositivos que interaccionen de forma fiable y segura con el sistema nervioso parece cada vez mas cerca. Sin embargo, aunque pensamos que la investigación 
Nuevos dispositivos electrónicos para interaccionar...

científica está destinada a revolucionar la calidad de vida de muchos enfermos en las próximas décadas, creemos que es necesario avanzar poco a poco, considerar todas las posibilidades, y sobre todo no crear falsas esperanzas que pueden afectar de forma negativa la credibilidad de estas investigaciones.

Un elemento básico que tienen en común los diferentes tipos de neuroprótesis es su necesidad de interaccionar directamente con las células nerviosas. Por ello uno de los grandes retos de la neurobiología actual y de la investigación en sistemas neuroprostésicos es comprender mejor la estructura y el funcionamiento del sistema nervioso. Es importante considerar el procesamiento en paralelo de la información, ya que las aspiraciones de desarrollar dispositivos que imiten el funcionamiento de los seres vivos, parecen cada vez más ligadas a la posibilidad de implementar artificialmente el alto grado de paralelismo que impera dentro de los sistemas biológicos. Además es imprescindible tener en cuenta la plasticidad o capacidad que presenta el sistema nervioso, incluso el cerebro adulto, para remodelarse y adaptarse a cualquier nueva situación. Durante muchos años se consideró al sistema nervioso central como una estructura funcionalmente inmutable y anatómicamente estática (Bergado-Rosado y Almaguer-Melian, 2000). En este sentido, Ramón y Cajal escribió en su obra Degeneración y regeneración en el sistema nervioso:

«... la especialización funcional del cerebro impone a las neuronas dos grandes lagunas: incapacidad de proliferación e irreversibilidad de la diferenciación intraprotoplasmática. Es por esta razón, que una vez terminado el desarrollo, las fuentes de crecimiento y regeneración de los axones y dendritas se secan irrevocablemente. En los cerebros adultos las vías nerviosas son algo fijo, terminado, inmutable»

Pero Don Santiago no sería el Maestro si no hubiera escrito al final del párrafo:

"Corresponde a la ciencia del futuro cambiar, si es posible, este cruel decreto»

Este no es un concepto nuevo ni presenta un reto insoluble ya que con los implantes auditivos este mismo problema se ha resuelto satisfactoriamente. Las neuroprótesis auditivas codifican señales acústicas con un rango de frecuencias muchísimo más limitado que las bandas que somos capaces de diferenciar con nuestro oído, sin embargo con el entrenamiento adecuado y gracias a la plasticidad cerebral, los humanos sordos son capaces de adaptarse al nuevo mundo auditivo creado por la prótesis y funcionar como sujetos oyentes. Esta misma esperanza, traducida a otros 
contextos como el visual, existe y alienta el desarrollo de sistemas de rehabilitación que ayuden a mejorar las condiciones de vida de las personas que padecen estos problemas. Sin embargo existen todavía muchas cuestiones relacionadas con la biocompatibilidad, la seguridad y eficacia, e incluso cuestiones no tecnológicas (relacionadas por ejemplo con la aceptación de estos dispositivos por parte de los médicos y los pacientes y la determinación de quién debe pagar por ellos) que necesitan ser contestadas antes de que estas alternativas puedan ser consideradas como una opción terapéutica mas. Pensamos que el actual ritmo de avances tanto en experimentación animal como en el terreno tecnológico y de fabricación de materiales permitirá contestar a muchas de estas preguntas, especialmente si se invierten los recursos humanos y materiales necesarios.

En los próximos años, los avances en el conocimiento de muchas enfermedades y en diferentes disciplinas como la medicina, biología, genética, y farmacología, permitirán diseñar nuevas formas de prevenir y tratar estas enfermedades. Sin embargo las terapias genéticas y farmacológicas no podrán evitar muchos daños debidos a accidentes y probablemente no serán capaces de solucionar todos los problemas degenerativos y derivados del envejecimiento. Los avances en sistemas y tecnologías neuroprostésicas son por tanto una necesidad para el futuro y pueden llegar a representar una opción terapéutica mas, para mejorar la calidad de vida de muchas personas. La comprensión y el apoyo de la sociedad a estas investigaciones multidisciplinares, donde médicos, neurobiólogos, ingenieros y otros especialistas tienen que trabajar de forma coordinada y generosa, es también fundamental para el desarrollo de estas tecnologías.

\section{Agradecimientos}

Al Dr. Markus Bongard (Universidad Miguel Hernández), por su ayuda en la preparación de las imágenes que acompañan este texto.

\section{Notas}

1 Federal Drug Administration

\section{Bibliografía}

Badi A. N., Kertesz T. R., Gurgel R. K., Shelton C., Normann R. A. (2003): Development of a novel eighth-nerve intraneural auditory neuroprosthesis. Laryngoscope 113, 833-42. 


\section{Nuevos dispositivos electrónicos para interaccionar...}

Barbeau H., Mccrea D. A., O'Donovan M. J., Rossignol S., Grill W. M., Lemay M. A. (1999): Tapping into spinal circuits to restore motor function. Brain Res Brain Res Rev 30, 27-51.

BEDFord F. L. (1999): Keeping perception accurate. Trends Cogn Sci 3, 4-11.

Bergado-Rosado J. A., Almaguer-Melian W. (2000): Mecanismos celulares de la neuroplasticidad. Rev Neurol 31, 1074-1095.

Branner A., Stein R. B., Fernandez E., Aoyagi y, Normann R. A. (2004): Long-term stimulation and recording with a penetrating microelectrode array in cat sciatic nerve. IEEE Trans Biomed Eng 51, 146-57.

BRAZIER M. A. B. (1984). A history of neurophysiology in the 17th \& 18th centuries. Raven Press: New York, N.Y.

BRINDLEY G. S. (1982): Effects of electrical stimulation of the visual cortex. Hum Neurobiol 1, 281-283.

Brindley G. S., Donaldson P. E. K., Falconer M. A., Rusthon D. N. (1972): The extent of the region of occipital cortex that when stimulated gives phosphenes fixed in the visual field. J Physiol (Lond) 225, 57-58.

BRINDLEY G. S., LEWIN W. S. (1968): The sensations produced by electrical stimulation of the visual cortex. J Physiol (Lond) 196, 479-493.

BRODMANN K. (1909). Vergleichende Lokalisationslehre der Grosshirnrinde in ihren Prinzipien dargestellt auf Grund des Zellenbaues: Leizpig.

CAJAL S. R. (1904). Textura del sistema nervioso del hombre y de los vertebrados. Imprenta y librería de Nicolás Moya: Madrid.

CAJAL S. R. (1914). Estudios sobre la degeneración y regeneración del sistema nervioso: Madrid.

Calford M. B., Wright L. L., Metha A. B., Taglianetti V. (2003): Topographic plasticity in primary visual cortex is mediated by local corticocortical connections. J Neurosci $23,6434-42$.

Chow A. Y., Pardue M. T., Chow V. Y., Peyman G. A., Liang C., Perlman J. I., Peachey N. S. (2001): Implantation of silicon chip microphotodiode arrays into the cat subre tinal space. IEEE Trans Neural Syst Rehabil Eng 9, 86-95.

Cohen L. G., Celnik P., Pascual-Leone A., Corwell B., Falz L., Dambrosia J., Honda M., Sadato N., Gerloff C., Catala M. D., HalletT M. (1997): Functional relevance of cross-modal plasticity in blind humans. Nature 389, 180-3.

Delbeke J., Oozeer M., Veraart C. (2003): Position, size and luminosity of phosphenes generated by direct optic nerve stimulation. Vision Res 43, 1091-102.

Delbeke J., Wanet-Defalque M. C., Gerard B., Troosters M., Michaux G., Veraart C. (2002): The microsystems based visual prosthesis for optic nerve stimulation. Artif Organs 26, 232-4.

DOBELLE W. H. (2000): Artificial vision for the blind by connecting a television camera to the visual cortex. Asaio J 46, 3-9.

Dobelle W. H., Mladejovsky M. G. (1974): Phosphenes produced by electrical stimulation of human occipital cortex, and their application to the development of a prosthesis for the blind. J Physiol (Lond) 243, 553-576.

Dobelle W. H., Mladejovsky M. G., Evans J. R., Roberts T. S., Girvin J. P. (1976): 'Braille' reading by a blind volunteer by visual cortex stimulation. Nature 259, 111-112.

Dobelle W. H., Mladejovsky M. G., Girvin J. P. (1974): Artificial vision for the blind: electrical stimulation of visual cortex offers hope for a functional prosthesis. Science $183,440-444$ 
Donoghue J. P. (2002): Connecting cortex to machines: recent advances in brain interfaces. Nat Neurosci 5, 1085-8.

DuBOIS-REYMOND E. (1849). Untersuchungen über thierische Elektricität: Berlin.

ECKMILLER R. (1997): Learning retina implants with epiretinal contacts. Ophthalmic Res 29, 281-9.

Fernandez E., Alfaro A., Tormos J. M., Climent R., Martínez M., Vilanova H., Walsh V., PASCUAL-LEONE A. (2002): Mapping of the human visual cortex using image-guided transcranial magnetic stimulation. Brain Res Protocols 10, 115-124.

Fernandez E., Pelayo F., Ahnelt P., Ammermuller J., Normann R. A. (2004): Cortical visual neuroprosthesis for the blind. Restorative Neurology Neuroscience.

GALVANI L. (1791). De viribus electricitatis in motu musculari, commentarious.

Ghazanfar A. A., Stambaugh C. R., Nicolelis M. A. (2000): Encoding of tactile stimulus location by somatosensory thalamocortical ensembles. J Neurosci 20, 3761-75.

HARRIS C. S. (1965): Perceptual adaptation to inverted, reversed, and displaced vision. Psychol Rev 72, 419-44.

HEIDUSCHKA P., THANOS S. (1998): Implantable bioelectronic interfaces for lost nerve functions. Prog Neurobiol 55, 433-461.

Hillman T., Badi A. N., Normann R. A., Kertesz T., Shelton C. (2003): Cochlear nerve stimulation with a 3-dimensional penetrating electrode array. Otol Neurotol 24, 7648.

HodGKIN A. L. (1964). The conduction of the nervous impulse. Liverpool University Press: Liverpool.

HodGKIN A. L., HuXley A. F. (1952): A quantitative description of membrane current and its application to conduction and excitation in nerve. J Physiol 117.

Lakhanpal R. R., Yanai D., Weiland J. D., FujiI G. Y., Caffey S., GreenberG R. J., DE JUAN E., JR., HUMAYUN M. S. (2003): Advances in the development of visual prostheses. Curr Opin Ophthalmol 14, 122-7.

Laubach M., Wessberg J., Nicolelis M. A. (2000): Cortical ensemble activity increasingly predicts behaviour outcomes during learning of a motor task. Nature 405, 56771.

Loeb G. E., Richmond F. J. R. (2001). BION implants for therapeutic and functional electrical stimulation. En: Neural prostheses for restoration of sensory and motor function. Chapin JK, Moxon KA, (eds). CRC Press LLC: Boca Raton, Florida, pp 75-101.

Margalit E., SAdDA S. R. (2003): Retinal and optic nerve diseases. Artif Organs 27, 96374.

MaynaRd E. M. (2001): Visual prostheses. Annu Rev Biomed Eng 3, 145-68.

Maynard E. M., Fernandez E., Normann R. A. (2000): A technique to prevent dural adhesions to chronically implanted microelectrode arrays. J Neurosci Methods 97, 93-101.

Mussa-Ivaldi F. A., Miller L. E. (2003): Brain-machine interfaces: computational demands and clinical needs meet basic neuroscience. Trends Neurosci 26, 329-34.

NiCOLELIS M. A. (2001): Actions from thoughts. Nature 409, 403-7.

NiCOLELIS M. A. (2003): Brain-machine interfaces to restore motor function and probe neural circuits. Nat Rev Neurosci 4, 417-22.

Nicolelis M. A., ChaPIN J. K. (2002): Controlling robots with the mind. Sci Am 287, 46-53.

Normann R. A., Maynard E., Guillory K. S., Warren D. J. (1996): Cortical implants for the blind. IEEE Spectrum, 54-59.

Normann R. A., Maynard E. M., Rousche P. J., Warren D. J. (1999): A neural interface for a cortical vision prosthesis. Vision Res 39, 2577-87. 


\section{Nuevos dispositivos electrónicos para interaccionar...}

Normann R. A., Warren D. J., Ammermuller J., Fernandez E., Guillory S. (2001): High-resolution spatio-temporal mapping of visual pathways using multi-electrode arrays. Vision Res 41, 1261-75.

Ortega Y Gasset J. (1939). Meditación de la técnica. Revista de Occidente: Madrid.

Pascual-Leone A., Cammarota A., Wassermann E. M., Brasil-Neto J. P., Cohen L. G., HALLETT M. (1993): Modulation of motor cortical outputs to the reading hand of braille readers. Ann Neurol 34, 33-7.

Pascual-Leone A., Hamilton R., Tormos J. M., Keenan J. P., Catala M. D. (1999). Neuroplasticity in the adjustment to blindness. En: Neuronal Plasticity: Building a Bridge from the laboratory to the Clinic. J.Grafman, Christen Y, (eds). Springer-Verlag: Berlin Heidelber New York.

PENField W., Jaspers H. (1974). Epilepsy and the functional anatomy of the human brain. Churchill: London, England.

PENFIELD W., RASMUSSEN T. (1950). The cerebral cortex of man. Macmillan: New York.

RIENER R. (1999): Model-based development of neuroprosthesis for paraplegic patients. Philos Trans R Soc Lond B Biol Sci 354, 877-94.

Rizzo J. F., Wyatt J., Loewenstein J., Kelly S., Shire D. (2003): Methods and perceptual thresholds for short-term electrical stimulation of human retina with microelectrode arrays. Invest Ophthalmol Vis Sci 44, 5355-61.

Rousche P. J., NoRMANN R. A. (1999): Chronic intracortical microstimulation (ICMS) of cat sensory cortex using the Utah Intracortical Electrode Array. IEEE Trans Rehabil Eng 7, 56-68.

SchmidT E. M., BAK M. J., HAMbrecht F. T., Kufta C. V., O'Rourke D. K., VallabhaNATH P. (1996): Feasibility of a visual prosthesis for the blind based on intracortical microstimulation of the visual cortex. Brain 119, 507-22.

Sekiyama K., Miyauchi S., Imaruoka T., Egusa H., Tashiro T. (2000): Body image as a visuomotor transformation device revealed in adaptation to reversed vision. Nature 407, 374-7.

Veraart C., Wanet-Defalque M. C., Gerard B., Vanlierde A., Delbeke J. (2003): Pattern recognition with the optic nerve visual prosthesis. Artif Organs 27, 996-1004.

WARING M. D. (1996): Properties of auditory brainstem responses evoked by intra-operative electrical stimulation of the cochlear nucleus in human subjects. Electoenceph Clin Neurophysiol 100, 538-548.

Warren D. J., Fernandez E., Normann R. A. (2001): High-resolution two-dimensional spatial mapping of cat striate cortex using a 100-microelectrode array. Neuroscience $105,19-31$.

ZRENNER E. (2002): Will retinal implants restore vision? Science 295, 1022-5.

Zrenner E., Stett A., Weiss S., Aramant R. B., Guenther E., Kohler K., Miliczek K. D., Seiler M. J., Haemmerle H. (1999): Can subretinal microphotodiodes successfully replace degenerated photoreceptors? Vision Res 39, 2555-67. 